\title{
Influence of movement on quality of seniors' lives
}

Z. Hudakova (Zuzana Hudakova)1,2, T. Friediger (Teresa Friediger)'1,

L. Lysa (Ludmila Lysa) ${ }^{1}$, M. Dohnanska (Maria Dohnanska)',

M. Sutvajova (Miroslava Sutvajova) ${ }^{1}$, A. Lesnakova (Anna Lesnakova) ${ }^{1}$

${ }^{1}$ Faculty of Health, Catholic University in Ruzomberok, Slovakia.

${ }^{2}$ Polytechnic University in Jihlava, Czech Republic.

\section{E-mail address:}

zuzana.hudakova@ku.sk

\section{Reprint address:}

Zuzana Hudakova

Faculty of health, Catholic University in Ruzomberok

Physiotherapy Department

Nam. A. Hlinku 48

03401 Ruzomberok

Slovakia

Source: Clinical Social Work and Health Intervention

Pages: $71-77$

Volume: 11

Issue: 3

\section{Reviewers:}

Steve Szydlowski

University of Scranton School of Education, USA

Gabriela Lezcano

San Francisco, CA, USA

\section{Keywords:}

Movement. Quality of Life. Seniors. Kinesiotherapy.

\section{Publisher:}

International Society of Applied Preventive Medicine i-gap

CSWHI 2020; 11(3): 71 - 77; DOI: 10.22359/cswhi_11_3_11 (C) Clinical Social Work and Health Intervention

\section{Abstract:}

Nowadays the average age of population is increasing. The society looks for bases which would allow the seniors to be independent within their limits as long as possible and to live dignified with the highest level of quality of their lives. Actually, there are not a lot of possibilities for movement activities under professional supervision for seniors in Slovakia. We observe their deficit in communities on the level of towns and villages and also in the organizations of social services or establishments for seniors. The aim of our work was to find out if we can achieve increments of quality of life among seniors by regular kinesiotherapy under observation of an expert. Stat- 
ing the hypothesis, we assigned the zero and the alternative hypothesis on the base level of significance $\alpha=0.05$. We divided the respondents into two groups. Both groups were comparable on the base of values of the test. In the group which completed the movement activities, more expressive improvement was achieved.

\section{Introduction}

It is extremely important to keep mobile in a higher age. Movement decreases the risk of cardio-vascular illnesses, diabetes, apoplectic stroke, obesity, osteoporosis; it improves breathing; it decreases cholesterol; it supports the correct activity of the heart. All these positives for movement preserve solid levels of quality of senior's lives. Quality of life is perceived subjectively as an evaluation of the human life expressed by feelings of happiness and satisfaction. They should be the result of influence and bilateral interaction of external (social, economical, environmental) and internal (psychological) realities on the human's life (Andrasko 2008). Topinkova (2005) states that health is one of the most important values of a human. Dutch Professor Veenhoven contributed to the categorization of the quality of life in his theory of Four Quadrants of Life. So allocated qualities of life admit to categorize concepts of a good life according to the aspect of the quality of life they are concerned with.

Quadrant A encloses advisability of the environment for the life and it emphases living, ecological, social, economical and cultural conditions.

Quadrant B is related to the concept of viability of an individual and we rate physical and psychical health into this group.

Quadrant C presents effectiveness of life and it is interested in how an effective individual contributes to the good of other people.

Quadrant D presents comprehension of quality of life and appreciation to life (Mares 2006).

Review of the quality of life is necessary when the aim of the treatment is previously established relative comfort of symptoms and keeping functional capacities, especially independence. (Kociova, Peregrinova 2003)

\section{Kinesiotherapy}

Treatment through movement has a consider- able influence on seniors' lives. By the help of kinesiotherapy we try to keep maximal self-sufficiency of seniors. Besides the application of the movement treatment we aim at psychological, social, as well as economic areas of these persons (Musilova, Masaryk, Mackinova 2014). Positive consequences of movement activity for senior's lives are shown because they allow an increase in physical abilities of patients. By creation of socalled energetic allowance it is possible to cope also with casual more demanding movement activities which increases the possibilities of social exertion for a senior (Pysna et al. 2009, Macek, Mackova 2008).

Many studies prove the positive influence of movement activity on the quality of life and that also is applicable for seniors. Positive changes for their functional and health state with the improvement or maintaining functions of their bodies are documented. Among the most frequent illnesses which we can influence by the help of kinesiotherapy belong atherosclerosis, diabetes mellitus, obesity \&/or osteoporosis (Hegyi,L 2012)

\section{Methodology of the work}

The aim of our work was to find out if we can achieve an increasing of quality of seniors' lives through regular kinesiotherapy. At the point of the articulation of the hypothesis we have defined the zero and alternative hypothesis on the base line of significance $\alpha=0.05$.

Hypothesis 1. H0: After the application of kinesiotherapy the status of patients didn't change. Kinesiotherapy doesn't influence the quality of senior's life.

H1: After the application of kinesiotherapy the status of the patients has been changed. Significant improvement of their mobility judged by the Barthel's test has occurred. We impute increasing of values in the Barthel's test to the influence of kinesiotherapy.

2. H0: The age doesn't influence the values of the Barthel's test in case of monitored respondents. 
H1: Age influences the values of the Barthel's test. The value of the Barthel's test decreases with the growing age of the respondents.

3. H0: We anticipate that quality of life isn't dependent on mobility and self-sufficiency of a senior.

H1: We anticipate that quality of senior's life is dependent on mobility and self-sufficiency of a senior for more than $60 \%$.

4. H0: Quality of seniors' lives in monitored establishments is the same. There is no difference in the quality of seniors' lives in monitored establishments.

H1: Quality of seniors' lives in monitored establishments is markedly different. Quality of seniors' lives in the establishment with the physiotherapy is markedly higher than in the establishment where the physiotherapy doesn't work.

As a method of the research we chose the test of the quality of life after Spitzer and the Barthel's index for monitoring of mobility and self-sufficiency of the patient. The test of the quality of life after Spitzer for reviewing of quality of life in the old age consisted of 5 points where: 1) activity; 2) self-sufficiency; 3 ) feeling of health; 4) family support; 5) allover feeling of satisfaction were reviewed. The point evaluation 2.1 and 0 were assigned to each question, whereby the number 2 represented a good evaluation, it means that the patient was active and self-sufficient; he/she felt well; had family support; was globally satisfied; balanced. On the contrary in the evaluation by the number 0 the patient wasn't active; he/she didn't handle the self-care; , he/she felt badly; was weak and tired out. The Barthel's index for monitoring of mobility and self-sufficiency of the patient observed 10 basic activities: 1) eating/drinking; 2) dressing; 3) bathing; 4) personal hygiene; 5) urine continence; 6) stool continence; 7) using the toilet; 8) transfer from the bed onto a chair; 9) walking on the flat ground; 10) going downstairs and upstairs. Each activity was evaluated after the fact if the patient copes with it himself/herself or with the help or if he/she doesn't cope with it at all. The maximal point evaluation was 100 points and it represented the independence of the patient and his/her full self-sufficiency. The minimal evaluation was 0 and it represented the high dependence of the patient. The evaluation of the grade of dependence after the achieved point evaluation was in the range 0-40 which represented the high dependence; 45-60 represented the medium grade dependence; 65-95 points meant mild dependence and 100 points represented full independence of the patient.

Characteristics of the research sample. The research took place in two establishments for seniors in the Slovak Republic in 2019. The first establishment was the experimental; the second was the control. In both, 30 respondents were engaged in the research; altogether 60 in the ages 65-93 with various needs of the client with the adaption for his/her health status. At the entry examination we defined the value of the Barthel's test and consequently after the completion of the research we administered the Barthel's test again. We divided them also by sex and we observed the measure of value influencing of the Barthel's test. In the control establishment there were respondents without kinesiotherapy and we used these results of the Barthel's test which were measured after the entry to the establishment and the second measure was done during our research and then we compared them.

Methods of elaboration of results. We elaborated results of the research statistically in the program MS EXEL. For the graphical figuration, column and line graphs were used. For the elaboration of results, particular elements from the data analysis - descriptive statistics, coloration were used. For the verification of hypothesis we used the binate T-test of two medium values on the significant level 0,05 and the F-test of two dispersions.

\section{The first hypothesis.}

In the first, experimental establishment, there were 19 women who represented $63.3 \%$ at the average age of 80 years. There were 11 men, with a means $36.7 \%$ at the average age of 78 years. The average age of all respondents was 79 years. The clients were evaluated by the Barthel's test before the start of kinesiotherapy and after the finish. We observed the difference between the final and the entry value of the Barthel's test. We worked with the clients for 3 months 3 times a week for 30 minutes. The aim was to find out if the improvement of mobility and self-sufficient activities occurs by kinesiotherapy in case of observed patients. 
Table 1 Barthel test values before and after kinesiotherapy in an experimental facility

\begin{tabular}{|l|c|c|c|c|}
\hline & \multicolumn{4}{|c|}{ Average values } \\
\hline $\mathbf{n}$ & Age & $\begin{array}{c}\text { Barthel } \\
\text { test } \\
\text { before }\end{array}$ & $\begin{array}{c}\text { Barthel } \\
\text { test } \\
\text { after }\end{array}$ & Change \\
\hline 30 & 79 & 45.5 & 56.33 & 10.83 \\
\hline
\end{tabular}

Table 1 represents the average point value of the Barthel's test before the kinesiotherapy, after the realization of kinesiotherapy and the last data observes the average value of improvement after the realization of kinesiotherapy in points. On the basis of our calculations we have found out that the critical value in both cases of one sided and bilateral alternative hypothesis was lower than the value of the testing criterion $t$ stat $=4.387>1.699$ $/ 2.045 /=\mathrm{t}$.krit . Because the statistical value $\mathrm{t}$ stat is higher than the critical $t$ krit we recommend to admit the alternative hypothesis $\mathrm{H} 1$. The binate $\mathrm{t}$ - test affirmed us the assumption of the alternative hypothesis. In case of respondents there was a considerable improvement for more than 10 points of the evaluating scale of the Barthel's test after the realization of kinesiotherapy and we consider it as eminent.

In the second control establishment there were 14 women who represented $46.7 \%$ with the average age of 83.3 years. There were 16 men, representing $53.3 \%$ an average age of 81.6 years. The average age of all respondents was 82.4 years. In the first establishment respondents exercised with the physiotherapist, in the second establishment they didn't exercise with the physiotherapist and two control measurements were done there. The first measurement contained accessible data about the Barthel's test at the arrival of the senior into the establishment. The second measurement was accomplished by us in this research (Tab. 2).
Table 2 Barthel test values on arrival at the facility and current status in the control facility

\begin{tabular}{|l|c|c|c|}
\hline & $\begin{array}{c}\text { Barrel } \\
\text { test on } \\
\text { arrival }\end{array}$ & $\begin{array}{c}\text { Bartel's test } \\
\text { in this } \\
\text { research }\end{array}$ & Change \\
\hline Men & 67.81 & 48.12 & -19.68 \\
\hline Women & 62.82 & 54.64 & -8.21 \\
\hline Together & 65.5 & 51.17 & -14.33 \\
\hline
\end{tabular}

Globally, all respondents had an average value of the Barthel's test at the arrival into the establishment 65.5 points represented the upper limit of only mild dependence of the client. During our control measurements respondents achieved the average value of the Barthel' so test only 51.167 characterizing to a large extent a second grade of dependence, it means the medium grade dependence. Globally, the respondents achieved at an average worsening of 14.33 points. We can state that worsening of mobility and self-sufficient activities expressed by the Barthel's test occurred.

\section{The second hypothesis.}

In case of respondents we could observe if the age is a significant element in values of the Barthel's test. The graphic interpretation of the relative age and improvement on the scale of the Barthel's test is presented in the graph 1 .

\section{Graph 1 Barthel test values before and after kinesiotherapy in an experimental facility}

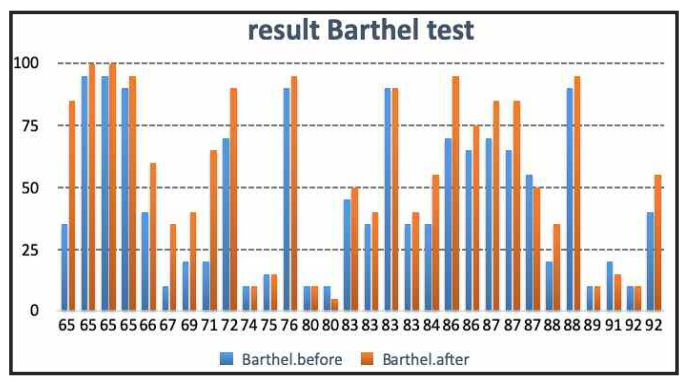

In Graph 1 values of the Barthel's test before the start of kinesiotherapy are presented by the blue color and after completing of kinesiotherapy by the red color. In the graph there are two distinct groups of respondents: the age of 65-80 years; $80-92$ years. Beside the age, the willingness to move actively is conditioned also by the sex of a respondent. 
Graph 2 Interpretation of the respondents' age and of the improvement on the scale of the Barthel's test in the experimental establishment

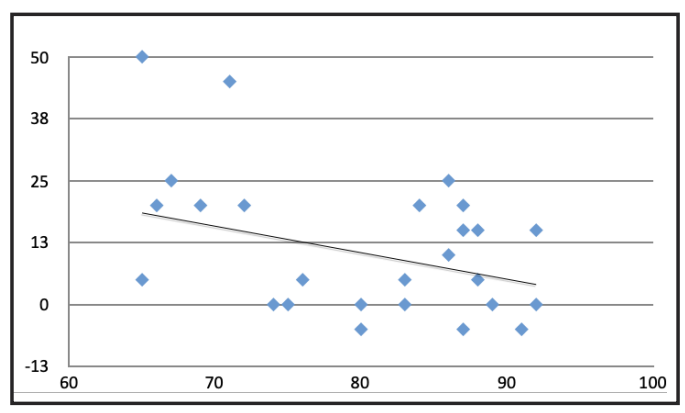

In Graph 2 we can clearly see that improvement of the respondents evaluated by the Barthel's test is conditioned by increasing age; measure of improvement degrees by the increasing age.

Graph 3 Values of the Barthel's test in the control establishment

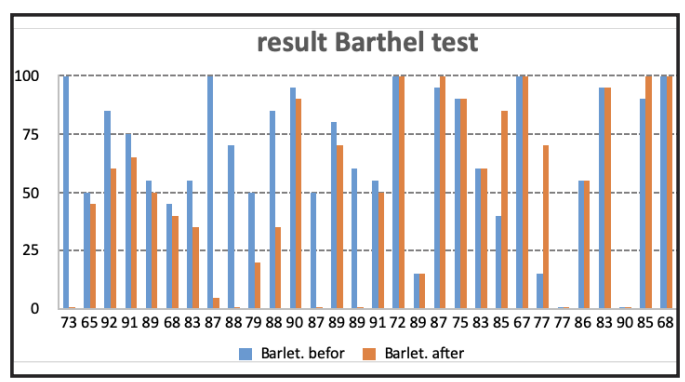

In the control establishment where respondents didn't accomplish kinesiotherapy, the values of the Barthel's test at the clients' arrival into the establishment are depicted by the blue color and the value of the Barthel's test during completing of our research is depicted by the red color. Most of respondents achieved decrease of values of the Barthel's test which evaluates clients' mobility and self-sufficiency in comparison with the values at the arrival to the establishment.

\section{The third hypothesis}

In another hypothesis we have assumed that quality of life is conditioned by mobility and selfsufficiency for more than $60 \%$. For approval of the hypothesis we used the Pearson's coefficient and the results are stated in the Table 3.
Table 3 Relationship between Barthel test and Spitzer quality of life test

\begin{tabular}{|l|l|l|l|}
\hline & $\begin{array}{c}\text { Barthel } \\
\text { test }\end{array}$ & $\begin{array}{c}\text { Spitzer } \\
\text { test }\end{array}$ & Pearson \\
\hline 2. zariadenie & 54.66667 & 65 & 0.845434 \\
\hline 1. zariadenie & 51.16667 & 59.33333 & 0.970829 \\
\hline
\end{tabular}

From the result of the Pearson's coefficient accrues the validity of Hypothesis H1. Achieved values are considerably higher than the assumed $60 \%$. In the experimental establishment where respondents accomplished kinesiotherapy, self-sufficiency of seniors improved in some cases up to $97 \%$. It accrues from this fact that the quality of senior's life is expressly also influenced by mobility and self-sufficiency of the senior. Relation between the Spitzer's test of quality of life and the Barthel's test in the experimental establishment is graphically depicted in the Graph 3 .

\section{Graph 4 Relation between the Spitzer's test and the Barthel's test in both estab- lishments}

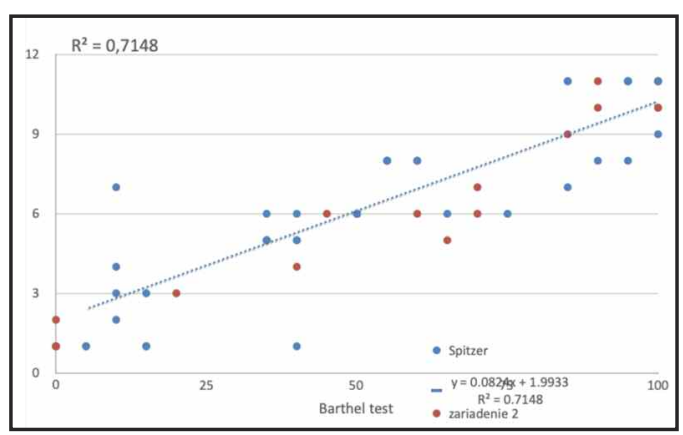

In the Graph 4 red points express the control establishment where respondents were without kinesiotherapy and blue points express the experimental establishment where respondents accomplished kinesiotherapy. We have affirmed that the senior's mobility and possibility to do self-sufficient activities evaluated by the Barthel's test influence directly the quality of life evaluated by the Spitzer's test.

\section{The fourth hypothesis.}

We observed if the quality of life is the same in both monitored establishments. For the declared hypothesis we used the binate $t$ - test of two medium values on the base level of significance $\alpha=0.05$. We indicate the result of the program EXCEL in the Table 4. 
Table 4 T-test of two mean values

\begin{tabular}{|l|l|l|}
\hline \multicolumn{3}{|c|}{$t$-Test: Two-Sample Assuming Equal Variances } \\
\hline$\alpha=0,05$ & Spitzer1 & Spitzer2 \\
\hline Mean & 6.5 & 5.933333 \\
\hline Variance & 9.982759 & 13.71954 \\
\hline Observations & 30 & 30 \\
\hline Pooled Variance & 11.85115 & \\
\hline $\begin{array}{l}\text { Hypothesized } \\
\text { Mean Difference }\end{array}$ & 0 & \\
\hline$d f$ & 58 & \\
\hline$t$ Stat & 0.637519 & \\
\hline$P(T<=t)$ one-tail & 0.263148 & \\
\hline$t$ Critical one-tail & 1.671553 & \\
\hline$P(T<=t)$ two-tail & 0.526296 & \\
\hline$t$ Critical two-tail & $\mathbf{2 . 0 1 7 1 7}$ & \\
\hline
\end{tabular}

The statistical value is considerably lower than the critical $\mathrm{t}$ stat $=0.63<1.67 / 2.00 /=\mathrm{t}$ krit . It emerges from this fact that the hypothesis $\mathrm{HO}$ was affirmed. Quality of seniors' lives is the same in both establishments. Hypothesis H0 was confirmed on the base level of significance $\alpha=0.05$. For our interest we increased our level of significance $\alpha=0.1$ and 0.25 , but also in this case our assume of the hypothesis H1 wasn't confirmed. Quality of life in the experimental establishment wasn't higher in comparison with the control establishment. Hypothesis H1 was confirmed upto the significance level higher than 0.25 which means an inaccessible failure from the point of view of applicability in practice.

\section{Discussion}

Affirming British research we can confirm that health problems grow with the age whereby the health measure in the youth was kept till the age of 50 years and then it decreased (Kind, 2008). Results of the research from 2015 published in Physiotherapia Slovaca confirm the fact that the subjectively perceived quality of life is significantly influenced through movement activities (Czarkowska, 2018). In Denmark there is the Danish Care Center. Seniors in this center get complex care. The outdoor and also indoor therapy is provided, they practice household care; they train walking an; they provide lingual therapy services (https://danishcarecenter.com). In
Germany so called mobile geriatric physiotherapy is performed. It means in practice that the necessary physiotherapy is provided at patients' homes by physiotherapists. They describe the main advantage that the patient is in his/her own home environment and he/she needn't travel to the physiotherapeutic center (https://www.evangelisches-johannesstift.de). The main principle of the German politics in the seniors' care consists not only in long-lasting care, but also in the need to carry physiotherapy into practice at the earliest . Also in Germany, geriatric clinics work for that purpose. In neighboring Austria, home care is expressively used during last years. The care of patients in the home environment has its advantages. Besides nursing and social help also other forms are used which are in the competence of the Red Cross and municipality, most frequently in the organization , Volkshilfe - national help ". If it is necessary the state contributes to the 24 hours long care by the needful accession where the family member can afford a home nurse or the needful physiotherapy. The quality of patient's life is improved by being in the home environment and by keeping a regular contact with his/her family and also his/her psychical status improves. The main principle of the German politics in the seniors' care consists not only in a long-lasting care, but also in the need to carry first physiotherapy into practice (Hudakova, Majernikova, 2013). In Germany women live on average for 81 years and men for 76 years of age.

\section{References:}

1. ANDRASKO I (2008) XI. International Colloquium on Regional Sciences. Brno: Masaryk University, Faculty of Economics and Administration, 2008. ISBN 9788021 046252.

2. CZARKOWSKA M (2018) Evolution of comprehensive ambulatory rehabilitation of people over 60 years of age available:https:// content.sciendo.com/view/journals/pjph/128/ 4/article-p144.xml.

3. HEGYI L (2012) Social gerontology 1. Ed.. Bratislava: Herba, 2012, p. 171. ISBN 97880-89171-996.

4. HUDAKOVA A, MAJERNIKOVA L (2013) Quality of life of a senior in the context of nursing. Grada Publishing as. 2013 Praha, ISBN 978-80-247-4772-9, p.128. 
5. KIND P et al. (2008) Variations in population health status. Results from a United Kingdom national questionnaire survey. Br. Med. J. p. 316, 736-741.

6. KOCIOVA K, PEREGRINOVA Z (2003) Physiotherapy in geriatrics, Osveta Martin 2003, ISBN 80-8063-132-8, p. 63.

7. LYSA L (2010) Statistics for managers, VERBUM Ruzomberok 2010, p. 159 ISBN 978-80-8084-609-1.

8. MACEK M, MACKOVA J (2008) The need for physical activity in old age. Med. Sport. Bohem. Slov, 2008,17,1, p. 34-42.

9. MARES J (2006) Prolems with the concept of quality of life and with its definition. Quality of life in children and adolescents. Brno: MSD, 2006. p. 13.
10. MUSILOVA E, MASARYK V, MAAKINOVA M (2014) Complex physiotherapy of muscle dysbalance at the upper cross syndrome. In: Medical Horizon. - Vol. 63, no. 12 (2014), p. 463-465. - ISSN (print) 0457-4214.

11. PYSNA J et al. (2009) Senior quality of life and exercise, Infopress 2009, ISBN 978-8085402-98-8 p. 171.

12. TOPINKOVAE (2005) Geriatric in practice. Praha: Galen, 2005 ISBN 80-7262-365-6.

13. https://www.evangelisches-johannesstift.de/ wichernkrankenhaus/mobile-geriatrische-rehabilitation.

14. https://danishcarecenter.com/therapy/?fblid =IwAR0aSCtsybm0YUJ-opLhw08-v8BSLI J8mUELCgCOexZ_DFqjoogjzHGT—o. 